\title{
EFFICIENT CFD/CSD COUPLING METHODS FOR AEROELASTIC APPLICATIONS
}

\author{
LONG CHEN* ${ }^{*}$ and XU TIANHAO \\ Department of Aerodynamics, Nanjing University of Aeronautics and Astronautics, \\ Nanjing, 210016, China \\ *Ichen@nuaa.edu.cn \\ XIE JING \\ Research \& Development Center, China Academy of Launch Vehicle Technology \\ Beijing, 100076, China \\ xiejing1024@126.com
}

Published 22 June 2016

\begin{abstract}
A fast aeroelastic numerical simulation method using CFD/CSD coupling are developed. Generally, aeroelastic numerical simulation costs much time and significant hardware resources with CFD/CSD coupling. In this paper, dynamic grid method, full implicit scheme, parallel technology and improved coupling method are researched for efficiency simulation. An improved Delaunay graph mapping method is proposed for efficient dynamic grid deform. Hybrid grid finite volume method is used to solve unsteady flow fields. The dual time stepping method based on parallel implicit scheme is used in temporal discretization for efficiency simulation. An approximate system of linear equations is solved by the GMRES algorithm with a LU-SGS preconditioner. This method leads to a significant increase in performance over the explicit and LU-SGS implicit methods. A modification of LU-SGS is proposed to improve the parallel performance. Parallel computing overs a very effective way to improve our productivity in doing $\mathrm{CFD} / \mathrm{CFD}$ coupling analysis. Improved loose coupling method is an efficiency way over the loose coupling method and tight coupling method. 3D wing's aeroelastic phenomenon is simulated by solving Reynolds-averaged Navier-Stokes equations using improved loose coupling method. The flutter boundary is calculated and agrees well with experimental data. The transonic hole is very clear in numerical simulation results.
\end{abstract}

Keywords: Fluid structure interaction; aeroelastic; dynamic grid; parallel.

\section{Introduction}

Accurate numerical simulation of aeroelastic is a complex and challenging multidisciplinary problem. Computational fluid dynamics requires accurate capabilities for modeling unsteady, three dimensional flowfields, transonic flow with shocks, fast grid deformation and efficient solve methods. This must be coupled with computational structural dynamics analysis for aeroelastic application. Over the past decade, there are several states of the art Reynolds-averaged Navier-Stokes (RANS) solvers are used in

This is an Open Access article published by World Scientific Publishing Company. It is distributed under the terms of the Creative Commons Attribution 3.0 (CC-BY) License. Further distribution of this work is permitted, provided the original work is properly cited. 
aeroelastic analysis such as CFL3D, TAU. Recently, some authors have presented and applied a coupled CFD and CSD code method for comprehensive analysis ${ }^{1-3}$.

Production level numerical simulations of aeroelastic generally involve complex geometries. The ability to handle complex geometries dictates consistent growth in grid sizes. At the same time, hardware parallelism has expanded dramatically such that simulations using thousands of cores are common today. Competitive solvers must be able to converge efficiently and robustly run parallelize ${ }^{4}$. Parallel computing overs a very effective way to improve our productivity in doing CFD/CFD coupling analysis. Another way to reduce turn-around time is to use full implicit methods. The objective of this work is to couple a CFD code with a CSD code in order to make progress in improving aeroelastic simulation efficiency.

\section{Computational Fluid Dynamics Solver}

An unstructured grid finite volume RANS solver was developed for present analysis ${ }^{5}$. The solver employs an implicit upwind algorithm in which the inviscid fluxes are obtained with HLLC Riemann solver second-order reconstruction based on the values and gradients on either side of interface. A lower-upper symmetric Gauss-Seidel (LUSGS) operator according to Jameson and Yoon is applied with GMRES method to time integral, with 2rd order time-accuracy dual-time stepping method for temporal discretization. The preconditioned GMRES algorithm is described below.

$$
\begin{array}{cc}
\text { Initialization: } & v_{0}=R-A \Delta W_{0} ; \quad r_{0}:=P^{-1} v_{0} ; \quad \beta:=r_{0} \|_{2} ; \quad v_{1}:=r_{0} / \beta \\
01: & \text { for } j=1, m \\
02: & w_{j}=P^{-1} A \Delta W \\
03: & \text { for } i=1, j \\
04: & \quad h_{i j}=\left(w_{j}, v_{i}\right) \\
05: & \quad w_{j}=w_{j}-h_{i j} v_{i} \\
06: & \text { end } \\
07: & h_{j+1, j}=\left\|w_{j}\right\|_{2} \\
08: & v_{j+1}=w_{j} / h_{j+1, j} \\
09: & z_{1: j}:=\min _{z}\left\|\beta e-H_{1: j} z_{1: j}\right\|_{2} \\
10: & \text { if }\left\|\beta e-H_{1: j} z_{1: j}\right\|_{2}<\varepsilon \text { exit } \\
11: & \text { end } \\
12: & \Delta W=\Delta W_{0}+v_{m} z_{m} \\
13: & \Delta W_{0}=\Delta W \\
14: & \text { restart gmres }
\end{array}
$$

Note that in the implementation of GMRES the least squares problem is not solved after the restart process but on the fly during the iteration(line 10 in equation 1 ) which be 
named MGMRES. Due to this reason, we have not performed every iteration to reach truncation criterion. This method can save computational time.

The use of GMRES combined with LU-SGS preconditioning techniques is becoming widespread in the CFD community for the solution of Navier-Stokes equations. A modification of $\mathrm{LU}_{-\mathrm{SGS}}{ }^{6}$ is proposed to improve the parallel performance. An approximate system of linear equations is solved by the GMRES algorithm with a parallel LU-SGS preconditioner. This method leads to a significant increase in performance over the explicit and LU-SGS implicit methods.

For turbulent flows, both the one-equation model of Spalart-Allmaras (SA) and the two-equation Menter's SST model are available. For all results presented in this paper, one equation SA model is employed.

\section{Delaunay Graph Mapping Method}

In the time-marching aeroelastic calculations, the grid must be updated at every time so that it conforms to the aeroelastically deformed shape of the structure. For unsteady computations, Dynamic grid based on Delaunay map ${ }^{5}$ is a fast, robust new algorithm. It has the characteristic of non-iterative, efficient and topological flexibility, avoid negative cell volumes.

A Delaunay graph of the whole grid is generated first. Insert auxiliary points around geometry to the initial Delaunay. Next, we map between the Delaunay graph and the computational grid during the movement. Delaunay graph can be moved easily during the geometric dynamic deformation, even for large deform. Therefore the new computational grid after the dynamic movement can be generated efficiently through the mapping while maintaining the primary qualities of the grid.

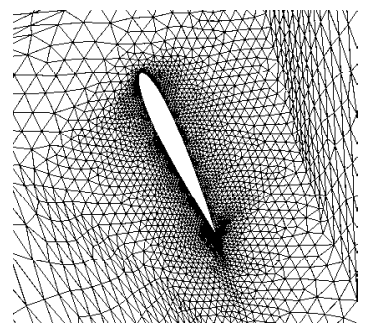

a. deformed grid

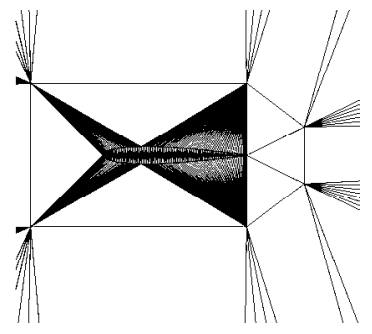

b. Delaunay graph

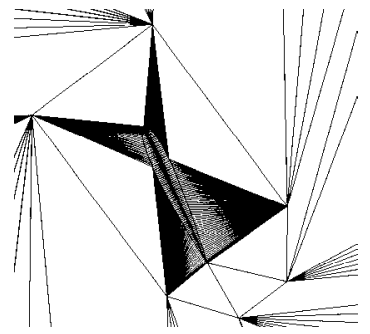

c. deformed Delaunay graph

Fig. 1. Improved Delaunay graph mapping method.

\section{Coupling Method}

In the loose coupling method, airloads data from the CFD solver and structure motion data from the CFD solver are exchanged at every unsteady time. The time step size must be very small to reserve time accuracy in the loose coupling method. In the tight coupling approach, the CFD and CSD codes are coupled at every inner time step, more grid deform and more FSI interpolation (TPS method) are needed. Improved loose coupling 
method $^{7}$ is an efficiency way over the loose coupling method and tight coupling method. Figure 2 shows flowchart of improved loose coupling method.

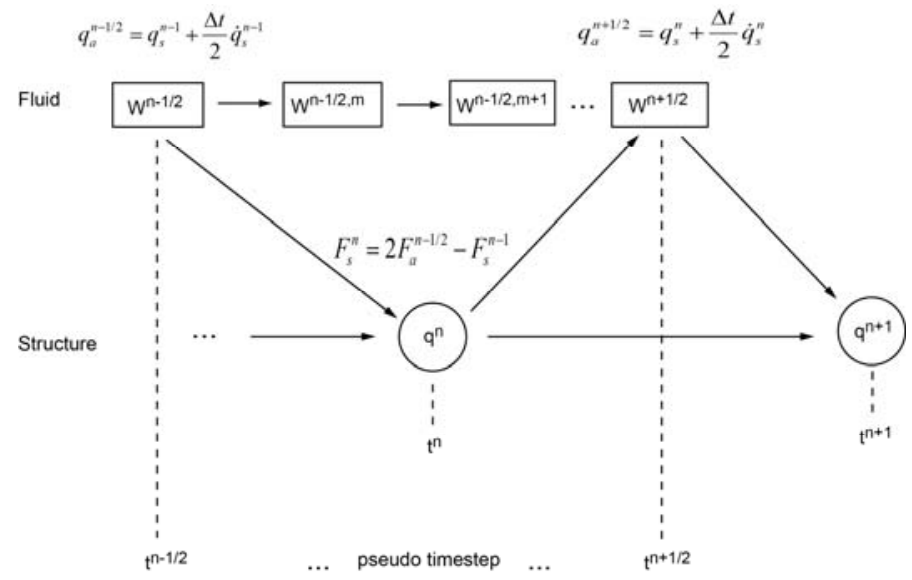

Fig. 2. Improved loose coupling method.

\section{Numerical Results}

The present efficiency method has been used to compute a variety of compressible flow problems for a wide range of conditions. We consider a turbulent flow over a wing-body configuration with Mach number 0.75 and $\mathrm{Re}=3.0 \cdot 10^{6}$. The test case is taken from the Drag Prediction Workshop (DPW) DLR-F6 configuration. The mesh is pure hexahedral and has about $5 \cdot 10^{6}$ points. Figure 3 and 4 display a comparison of convergence histories among the LU-SGS scheme, the GMRES scheme, the MGMRES scheme versus time step and CPU time, respectively. It is clear that MGMRES method is superior to LU-SGS method. The present MGMRES method is over 4 times faster than its LU-SGS counterpart.

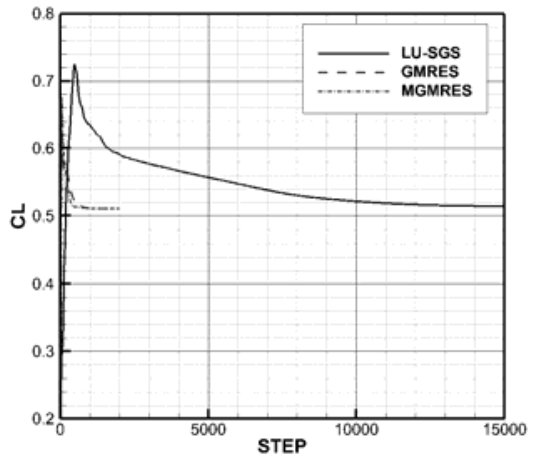

Fig. 3. Convergence rate versus step.

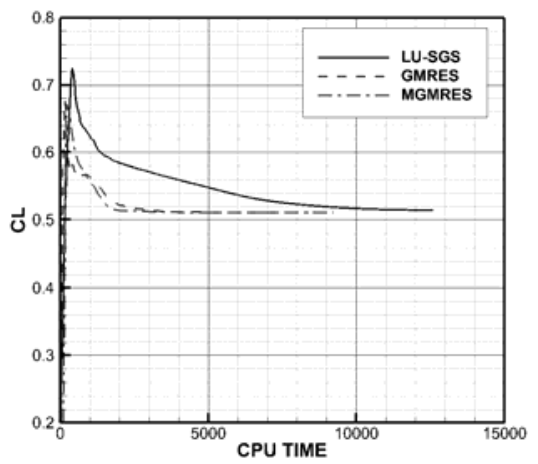

Fig. 4. Convergence rate versus CPU time. 
The speedup was measured by timing CPU time of one time step on different number of processors. The parallel speedup results reported in Figure 5 and 6 highlight the good parallel scalability of our simulation technology. Even more remarkable superlinear speedup is achieved on 240 processors.

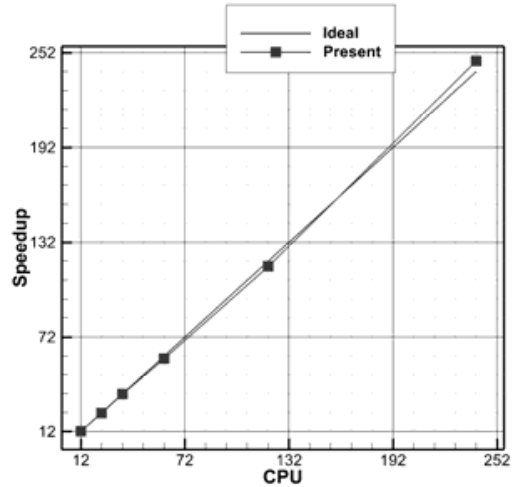

Fig. 5. Speedup for LU-SGS method.

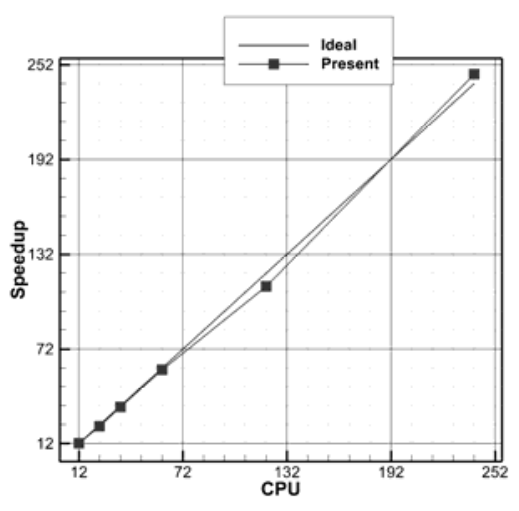

Fig. 6. Speedup for GMRES method.

The computed pressure coefficient distributions are compared with experimental data at six spanwise stations in Figures 7 . The results obtained compare closely with experimental data.

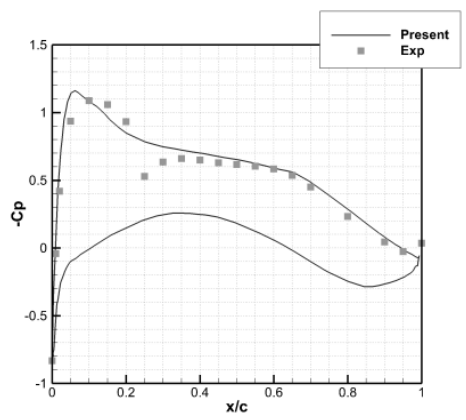

a. $\eta=15.0 \%$

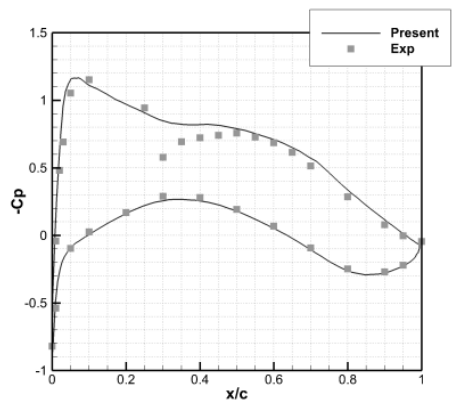

c. $\eta=51.4 \%$

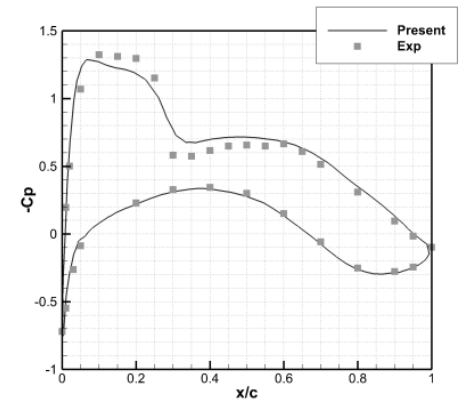

b. $\eta=33.1 \%$

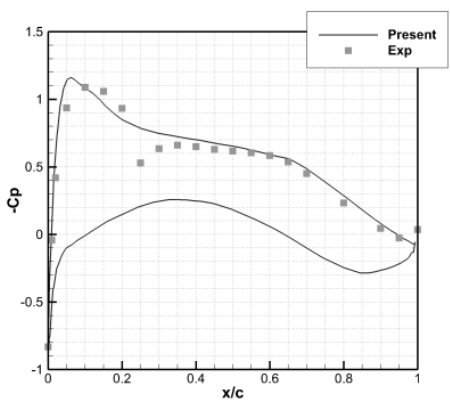

d. $\eta=84.7 \%$

Fig. 7. Pressure coefficient distributions. 
For validation of the present method for the unsteady flow computations, the numerical results are compared around the pitching NACA0012 airfoil. The free-stream Mach number is 0.755 . Figure 8 shows convergence histories with respect to LU-SGS, GMRES and MGMRES. The present MGMRES method is over 3 times faster than its LU-SGS counterpart.

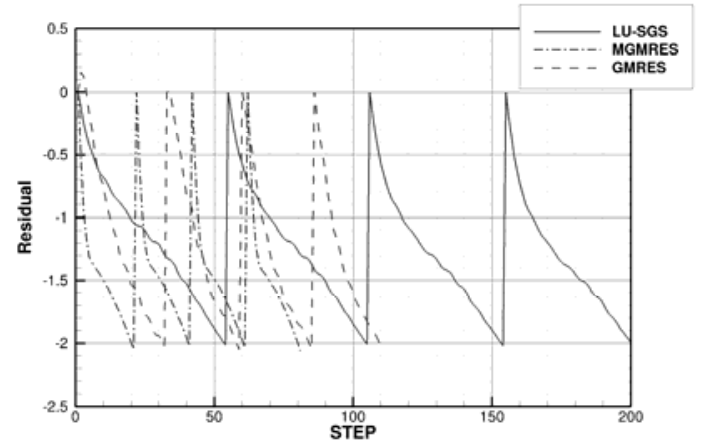

Fig. 8. Residual convergence rate for unsteady case.

We consider an aeroelastic problem over the AGARD 445.6 wing. We perform the computations on an unstructured mesh. Using present approach, we determined the flutter boundary of the weakened wing over the Mach number range 0.338-1.141.The flutter boundary is calculated and agrees well with experimental data. The transonic hole is very clear in numerical simulation results.

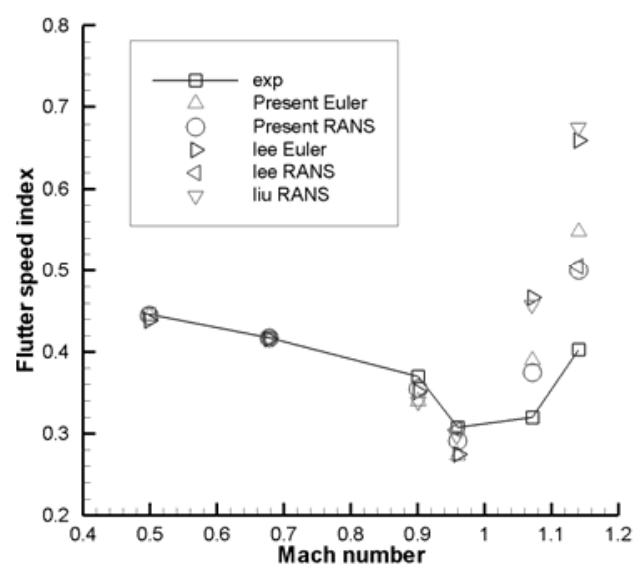

Fig. 9. Flutter speed index.

\section{Conclusions}

A parallel coupling CFD/CSD simulation code has been developed for the simulation and prediction of an aeroelastic system. The Robustness and fast convergence of the approach 
have been demonstrated. The numerical results obtained indicate that the use of the present method leads to a significant increase in performance.

\section{References}

1. R. Kamakoti and W. Shyy, Fluid-structure interaction for aeroelastic applications, Progress in Aerospace Sciences. 40, 535 (2004).

2. E. Lee-Rausch and J. T. Batina, Wing flutter boundary prediction using unsteady Euler aerodynamic method, AIAA Paper 1993-1422 (1993).

3. E. Lee-Rausch and J. T. Batina, Wing flutter computations using an aerodynamic model based on the Navier-Stokes equations, Journal of Aircraft. 33, 1139 (1996).

4. F. Liu, M. Sadeghi, S. Yang and H. Tsai, Parallel computation of wing flutter with a coupled Navier-Stokes/CSD method, AIAA Paper 2003-1347 (2003).

5. C. Long, W. Yizhao, X. Jian, Aeroelastic analysis of rotor blades using CFD/CSD coupling in hover, Modern Physics Letters B. 24, 1307 (2010).

6. A. M. Wissink, Parallelization of a three-dimensional flow solver for Euler rotorcraft aerodynamics predictions, AIAA Journal. 34, 2276 (1996).

7. C. Farhat and P. Geuzaine, Application of a three-field nonlinear fluid-structure formulation to the prediction of the aeroelastic parameters of an F-16 fighter, Computers \& fluids. 32, 3 (2003). 